\title{
Critical congenital heart disease screening
}

Mohammed A. Chamsi-Pasha, Hassan Chamsi-Pasha ${ }^{1}$

Division of Cardiovascular Medicine, University of Nebraska Medical Center, Omaha, Nebraska, USA, 'Division of Cardiovascular Medicine, King Fahd Armed Forces Hospital, Jeddah, Saudi Arabia

\begin{tabular}{|c|}
\hline Access this article online \\
\hline Website: www.avicennajmed.com \\
\hline DOI: 10.4103/2231-0770.184062 \\
\hline Quick Response Code: \\
\hline
\end{tabular}

ABSTRACT
Critical congenital heart disease (CCHD) is a heart lesion for which neonates require early surgical
intervention to survive. Without intervention, the rates of mortality and survival with significant
disability are extremely high. Early diagnosis can potentially improve health outcomes in newborns
with CCHD. Until recent years, no routine screening protocol existed. In the last few years, pulse
oximetry screening for CCHD in newborns has been added to the list of recommended uniform
screening panels and advocated by several health-care authorities. A positive screening test
result warrants an echocardiogram to evaluate for CCHD. Newborn screens do not usually require
parental consent. However, most of the states mandates in the United States include a statement
allowing exemption from the screen on the basis of parental religious or personal beliefs.
Key words: Critical congenital heart disease, pulse oximetry, screening

\section{INTRODUCTION}

Critical congenital heart defects are usually defined as structural malformations of the heart that are present at birth and require intervention in the $1^{\text {st }}$ year of life. ${ }^{[1]}$ Congenital heart disease has been reported to be responsible for $30-50 \%$ of infant mortality due to birth defects. ${ }^{[2]}$ During the period from 1999 to 2006 (before the advent of critical congenital heart disease [CCHD] newborn screening), more than 13,000 infant deaths caused by congenital heart defects were reported in the United States. ${ }^{[2]}$

Prenatal detection has become a significant contributor to early identification of CCHD. A recent meta-analysis has shown that newborns with a prenatal diagnosis of CCHD were significantly less likely to die before planned cardiac surgery than those with a comparable postnatal diagnosis. ${ }^{[3]}$ Even with fetal echocardiograms, which are not available universally, the diagnosis of CCHD during pregnancy is difficult. ${ }^{[4]}$

\section{SCREENING WITH PULSE OXIMETRY}

Early detection of CCHD is challenging; however, newborns screening using pulse oximetry has been strongly supported

Address for correspondence: Dr. Mohammed A. Chamsi-Pasha, Division of Cardiovascular Medicine, University of

Nebraska Medical Center, Omaha, Nebraska, USA.

E-mail: drpasha.moh@gmail.com by recent literature as a valuable tool facilitating the prompt detection of infants with CCHD. Pulse oximetry is easily accessible, inexpensive, and noninvasive and can be easily performed by the nurses at the infant's bedside; however, it remains a technology that is underutilized in newborns. ${ }^{[5]}$

In 2011, the US Secretary of Health and Human Services recommended adding screening for CCHD with pulse oximetry to the recommended uniform screening panel (RUSP). ${ }^{[6]}$

Screening programs for CCHD in the United States have been successful in identifying asymptomatic newborns with CCHD with few false positives. A pulse oximeter can help the nursery clinicians identify babies who have oxygen saturation levels below 95\% ( $\geq 95 \%$ is considered normal). Pulse oximetry sensitivity in detecting CCHD has been demonstrated to be high (76.5\%) with a specificity up to $99 \%$ with a very low false-positive rate, making it a strong test. ${ }^{[7]}$ In the Thangaratinam et al. meta-analysis, ${ }^{[8]}$ only de-Wahl Granelli et al. ${ }^{[9]}$ and Ewer et al..$^{[7]}$ studies used the currently

This is an open access article distributed under the terms of the Creative Commons Attribution-NonCommercial-ShareAlike 3.0 License, which allows others to remix, tweak, and build upon the work non-commercially, as long as the author is credited and the new creations are licensed under the identical terms.

For reprints contact: reprints@medknow.com

Cite this article as: Chamsi-Pasha MA, Chamsi-Pasha $\mathrm{H}$. Critical congenital heart disease screening. Avicenna J Med 2016;6:65-8. 
recommended algorithm. de-Wahl Granelli et al. ${ }^{[9]}$ showed a positive predictive value of $20 \%$ and Ewer et al. ${ }^{[7]} 13 \%$. This means that most of the babies who have negative screening test are unlikely to have CCHD.

When screening test is positive, follow-up investigations include echocardiograms and referrals to pediatric cardiologists. All infants with positive screens should be carefully assessed by well-trained pediatric staff. Hospitals need to have an established protocol regarding who and when to call for a positive screening. ${ }^{[10]}$

There is an increased interest in CCHD screening all over the world. It was estimated that $\geq 90 \%$ of infants born in the United States were screened for CCHD screening by the end of 2014. ${ }^{[1]]}$ Finland has the highest screening rate after implementation (97\%), followed by Sweden (91\%) and Norway (90\%). ${ }^{[12]}$ Researchers in China, the first low-income country to evaluate pulse oximetry screening for CCHD, found pulse oximetry screening in combination with clinical assessment to be reliable and feasible. ${ }^{[13]}$

\section{CLINICAL ASPECTS OF PULSE OXIMETRY}

Pulse oximetry is a widely available, accurate method to objectively quantify oxygen saturations $\left(\mathrm{SpO}_{2}\right)$, and thereby identify the clinically undetectable hypoxemia that occurs in the majority of neonates with CCHD. ${ }^{[14]} \mathrm{It}$ is recommended to use pulse oximeter devices that are cleared for use in newborns, are usable in low perfusion states, report functional oxygen saturation, and are motion tolerant.

\section{CUTOFF VALUES}

The definition of threshold values will determine the sensitivity and specificity of the screening tool. When choosing the cutoff value, the false positive rate must be balanced against the risk of missing CCHD. Ewer et al. defined $\mathrm{SpO}_{2}<95 \%$ in either limb or a difference of $>2 \%$ between the limbs as abnormal. ${ }^{[6,7]}$

\section{TEST ACCURACY}

The test accuracy can be influenced by gestational age, targets definition, timing of screening, and antenatal detection of CCHD. Early screening can lead to more false positive screenings but has the potential to detect significant pathology earlier. A meta-analysis of 13 screening studies, including almost 230,000 infants, reported a sensitivity of $76.5 \%$, specificity of $99.9 \%$, and false positive rate of $0.14 \% .{ }^{[8]}$
Sensitivity ranged from $60 \%$ to $100 \%$ whereas specificity was $\geq 94 \%$ and in most studies $>99 \%$. This high specificity is accompanied by a false positive rate varying between $0 \%$ and $1.8 \% \cdot{ }^{[15]}$

\section{DETECTION OF OTHER PATHOLOGIES}

Pulse oximetry can also detect other causes of hypoxemia, including infections and pulmonary/respiratory disorders. Noncritical cardiac defects and other significant pathologies may be found in up to $80 \%$ of the false positive cases. ${ }^{[7,9,13]}$

Although detection of these conditions is currently considered as false positives, it is important to detect them early so that treatment can be started before deterioration occurs with increased risk of death, morbidity, and longer hospitalization. There is large variation in detection of other pathologies in the reported studies $(0-90 \%){ }^{[15]}$

Bhola et al.$^{[16]}$ highlighted the benefits of early diagnosis of significant, noncardiac illness and proposed that a positive result is not an immediate trigger for echocardiography but rather should prompt careful, expert medical evaluation.

\section{TIMING}

Performing pulse oximetry screening in the $1^{\text {st }}$ hours of life is likely to lead to more false positive screenings, but this should be weighed against the potential benefit of detecting significant pathology, including noncritical CHD, infections, and pulmonary disorders, at an early stage of the disease, preventing deterioration. Screening performed $>24 \mathrm{~h}$ after birth decreases the false positive rate but increases the risk of late detection of infants with CCHD, who may decompensate before screening. ${ }^{[7,9]}$

The meta-analysis of Thangaratinam et al. ${ }^{[8]}$ demonstrated a significantly lower false positive rate when the screening was performed $\geq 24 \mathrm{~h}$ after birth. Ewer et al. ${ }^{[7]}$ showed the highest sensitivity if screening took place $6-12 \mathrm{~h}$ after birth, but specificity was the highest at $0-6 \mathrm{~h}$ after birth.

\section{SETTING}

In most countries where the pulse oximetry screening has been implemented, the screening takes place in hospitals. Pulse oximetry screening in the Neonatal Intensive Care Unit (NICU) has been less well investigated. Although screening in the NICU is feasible, underlying illnesses and timing of the screening increased the false positive rate. ${ }^{[17]}$ Studies have also investigated pulse oximetry screening out of hospital and after early discharge from hospital. An 
observational study showed that pulse oximetry can be successfully implemented outside the hospital setting. ${ }^{[18]}$ In a small study of ninety babies who underwent routine pulse oximetry screening within $2 \mathrm{~h}$ following homebirth, Cawsey et al. showed that early pulse oximetry screening for homebirths is both feasible and acceptable. ${ }^{[19]}$

\section{LIMITATIONS AND BARRIERS}

There are several limitations to pulse oximetry screening for CCHD in neonates. It does not exclude all types of CCHD and other congenital heart disease lesions. Knowing that the sensitivity of pulse oximetry screening for CCHD is around $75 \%$, approximately $25 \%$ of neonates with CCHD will not be detected by pulse oximetry screening alone. The critical lesions most likely to be missed by use of pulse oximetry are those that cause obstruction of the aortic arch (e.g., coarctation of the aorta and interrupted aortic arch). ${ }^{[7]}$ Hence, clinicians must be educated about these potential patients with false-negative results, so that other clinical findings of CCHD are not ignored. ${ }^{[20]}$

Important barriers to using pulse oximetry screening for CCHD have been identified. These include out-of-hospital births, cost, and knowledge deficits among health-care professionals. There are certainly costs and harms associated with both pulse oximetry screening and subsequent testing that ensues. In the United States, the direct cost of the screening itself is bundled into the hospital labor and delivery charge, so there is rarely a separate charge for oximetry to hospital-born babies. The echocardiograms triggered by positive oximetry test are additional charges that can be as high as thousands of dollars.

In addition to the anxiety triggered by a failed screening and the costs of additional screening, some babies may need to be transferred from their birth setting to another facility for echocardiography. The issue of transfer is particularly true in rural states where the nearest available echocardiography may be a great distance away.

Altitude, time, health status of newborns, and type of cardiac defect appear to affect results. All studies reported a maximum of 5.5 min per screening, with a mean of even 1.6 min in Zhao et al. study. ${ }^{[9,13,16]}$ No extra staff members were needed to perform the screening. ${ }^{[21,22]}$ Despite this, nurses have reported a minimal burden to their routine. Lower saturations at altitude are a unique barrier to the use of pulse oximetry for CCHD screening. Wright et al. ${ }^{[23]}$ found significantly higher failure rates at moderate altitude compared with sea level, suggesting alternative screening protocols for affected areas. Besides, there is a need to interpret the screening test using an algorithm. ${ }^{[24]}$ More training in implementing pulse oximetry screening may be particularly helpful for those nurses less familiar with this skill.

\section{ACCEPTABILITY}

Before administration, parents should be informed of the purpose of screening for CCHD along with other newborn screening procedures. Parents need to be informed of abnormal results and the follow-up process that will occur. ${ }^{[10]}$

Families that refuse pulse oximetry screening on religious grounds are exceptionally rare and generally are unrelated to the timing of symptoms.

The larger issue is the family that refuses additional testing once a baby has positive test results.

The objection to screening might be partly caused by the parents' desire not to know potential health diagnoses before they become symptomatic. This hurdle can be overcome with proper handling and explanation of the case. A recent online survey for 841 parents of children with congenital heart disease showed that the information given at diagnosis, the manner in which it is presented, and the parents' understanding and interpretation of that information are critical factors in shaping parents' perceptions and management decisions. ${ }^{[25]}$

A recent study reported that parents widely accepted the test and the false positive results did not lead to more anxiety. ${ }^{[26]}$ Furthermore, the medical staff considered the test as highly important and easy to carry out.

\section{CONCLUSION}

An accurate and early diagnosis of the type of CCHD has implications on the type of management of the disease. Pulse oximetry, a simple, inexpensive, noninvasive screening approach, can make the difference between a healthy life and life of disability or even death. Health policies need to ensure that all newborns receive the standard recommended pulse oximetry screening. Staff education is imperative before screening implementation. Pediatricians should be aware of the addition of pulse oximetry to screen for CCHD to the RUSP so that they can educate parents, ensure appropriate follow-up for positive screens, and stride for incorporating this screening in their local hospitals.

\section{Financial support and sponsorship}

Nil. 


\section{Conflicts of interest}

There are no conflicts of interest.

\section{REFERENCES}

1. Olney RS, Ailes EC, Sontag MK. Detection of critical congenital heart defects: Review of contributions from prenatal and newborn screening. Semin Perinatol 2015;39:230-7.

2. Gilboa SM, Salemi JL, Nembhard WN, Fixler DE, Correa A. Mortality resulting from congenital heart disease among children and adults in the United States, 1999 to 2006. Circulation 2010;122:2254-63.

3. Holland BJ, Myers JA, Woods CR Jr. Prenatal diagnosis of critical congenital heart disease reduces risk of death from cardiovascular compromise prior to planned neonatal cardiac surgery: A meta-analysis. Ultrasound Obstet Gynecol 2015;45:631-8.

4. Ailes EC, Gilboa SM, Riehle-Colarusso T, Johnson CY, Hobbs CA, Correa A, et al. Prenatal diagnosis of nonsyndromic congenital heart defects. Prenat Diagn 2014;34:214-22.

5. Amsbaugh S, Scott SD, Foss K. Pulse oximetry screening for critical congenital heart disease: Bringing evidence into practice. J Pediatr Nurs 2015;30:591-7.

6. Mahle WT, Martin GR, Beekman RH $3^{\text {rd }}$, Morrow WR; Section on Cardiology and Cardiac Surgery Executive Committee. Endorsement of health and human services recommendation for pulse oximetry screening for critical congenital heart disease. Pediatrics 2012;129:190-2.

7. Ewer AK, Middleton LJ, Furmston AT, Bhoyar A, Daniels JP, Thangaratinam $\mathrm{S}$, et al. Pulse oximetry screening for congenital heart defects in newborn infants (PulseOx): A test accuracy study. Lancet 2011;378:785-94.

8. Thangaratinam S, Brown K, Zamora J, Khan KS, Ewer AK. Pulse oximetry screening for critical congenital heart defects in asymptomatic newborn babies: A systematic review and meta-analysis. Lancet 2012;379:2459-64.

9. de-Wahl Granelli A, Wennergren M, Sandberg K, Mellander M, Bejlum C, Inganäs $\mathrm{L}$, et al. Impact of pulse oximetry screening on the detection of duct dependent congenital heart disease: A Swedish prospective screening study in 39,821 newborns. BMJ 2009;338:a3037.

10. Andrea J. Implications of using pulse oximetry to screen for critical congenital heart disease in newborns. Nurs Womens Health 2015; 19:314-23.

11. Hom LA, Martin GR. U.S. international efforts on critical congenital heart disease screening: Can we have a uniform recommendation for Europe? Early Hum Dev 2014;90 Suppl 2:S11-4.

12. de-Wahl Granelli A, Meberg A, Ojala T, Steensberg J, Oskarsson G, Mellander M. Nordic pulse oximetry screening - implementation status and proposal for uniform guidelines. Acta Paediatr 2014;103:1136-42.

13. Zhao QM, Ma XJ, Ge XL, Liu F, Yan WL, Wu L, et al. Pulse oximetry with clinical assessment to screen for congenital heart disease in neonates in China: A prospective study. Lancet 2014;384:747-54.

14. Dawson JA, Davis PG, O'Donnell CP, Kamlin CO, Morley CJ. Pulse oximetry for monitoring infants in the delivery room: A review. Arch Dis Child Fetal Neonatal Ed 2007;92:F4-7.

15. Narayen IC, Blom NA, Ewer AK, Vento M, Manzoni P, Te Pas AB. Aspects of pulse oximetry screening for critical congenital heart defects: When, how and why? Arch Dis Child Fetal Neonatal Ed 2016;101:F162-7.

16. Bhola K, Kluckow M, Evans N. Post-implementation review of pulse oximetry screening of well newborns in an Australian tertiary maternity hospital. J Paediatr Child Health 2014;50:920-5.

17. Manja V, Mathew B, Carrion V, Lakshminrusimha S. Critical congenital heart disease screening by pulse oximetry in a neonatal intensive care unit. J Perinatol 2015;35:67-71.

18. Lhost JJ, Goetz EM, Belling JD, van Roojen WM, Spicer G, Hokanson JS. Pulse oximetry screening for critical congenital heart disease in planned out-of-hospital births. J Pediatr 2014;165:485-9.

19. Cawsey MJ, Noble S, Cross-Sudworth F, Ewer AK. Feasibility of pulse oximetry screening for critical congenital heart defects in homebirths. Arch Dis Child Fetal Neonatal Ed 2016. pii: Fetalneonatal-2015-309936.

20. Bruno CJ, Havranek T. Screening for critical congenital heart disease in newborns. Adv Pediatr 2015;62:211-26.

21. Peterson C, Grosse SD, Glidewell J, Garg LF, Van Naarden Braun K, Knapp MM, et al. A public health economic assessment of hospitals' cost to screen newborns for critical congenital heart disease. Public Health Rep 2014;129:86-93.

22. Zuppa AA, Riccardi R, Catenazzi P, D’Andrea V, Cavani M, D'Antuono A, et al. Clinical examination and pulse oximetry as screening for congenital heart disease in low-risk newborn. J Matern Fetal Neonatal Med 2015;28:7-11.

23. Wright J, Kohn M, Niermeyer S, Rausch CM. Feasibility of critical congenital heart disease newborn screening at moderate altitude. Pediatrics 2014;133:e561-9.

24. Pattishall AE, Spector ND, McPeak KE. Important considerations for the newborn: Access to postdischarge newborn care, pulse oximetry screening for congenital heart disease, and circumcision. Curr Opin Pediatr 2014;26:734-40.

25. Hilton-Kamm D, Sklansky M, Chang RK. How not to tell parents about their child's new diagnosis of congenital heart disease: An Internet survey of 841 parents. Pediatr Cardiol 2014;35:239-52.

26. Powell R, Pattison HM, Bhoyar A, Furmston AT, Middleton LJ, Daniels JP, et al. Pulse oximetry screening for congenital heart defects in newborn infants: An evaluation of acceptability to mothers. Arch Dis Child Fetal Neonatal Ed 2013;98:F59-63. 